\title{
LASER COOLING OF RELATIVISTIC HEAVY ION BEAMS*
}

\author{
U. Schramm ${ }^{\dagger}$, M. Bussmann, D. Habs, LMU Munich, Germany \\ T. Kühl, K. Beckert, P. Beller, B. Franzke, F. Nolden, M. Steck, GSI, Darmstadt, Germany \\ G. Saathoff, S. Reinhardt, MPI-K, Heidelberg, Germany \\ S. Karpuk, Univ. Mainz, Germany
}

\begin{abstract}
We report on the experimental demonstration of laser cooling of a $C^{3+}$ ion beam performed at the ESR (GSI) at an energy of $E=1.47 \mathrm{GeV}$. The decelerating laser force of one Doppler-tuned UV laser beam was counteracted by moderately bunching the beam. This versatile scheme lead to longitudinally 'space-charge dominated' beams with an unprecedented momentum spread of $\Delta p / p \approx 10^{-7}$.

Concerning beam energy and charge state of the ion, the experiment depicts an important step from the field of laser cooling of ion beams at low energies toward the laser cooling scheme proposed for relativistic beams of highly charged heavy ions at the future GSI facility FAIR.
\end{abstract}

\section{THE PROSPECT OF LASER COOLING}

The cooling of stored heavy ion beams to high phasespace densities is of interest when low momentum spread or high luminosity is required. Ultimately, the reduction of the energy spread far below the mutual Coulomb energy of the ions leads to a phase transition - a crystallization of the ion beam - into a regime where collision dominated heating mechanisms vanish and maximum phase-space density is reached. In the low energy regime this phase transition could recently be demonstrated with laser-cooled ${ }^{24} \mathrm{Mg}^{+}$ ion beams in the rf quadrupole storage ring PALLAS $[1,3]$. However, as laser cooling relies on the repeated resonant scattering of photons, only a very limited number of ions can be accessed by this cooling technique at existing storage rings, benchmarking and present activities being summarized in [2].

At heavy ion storage rings like ESR (GSI), electron cooling has developed into a versatile tool for the cooling of highly charged ions independent from their internal atomic properties [4]. As the cooling force (and the inter-ion coupling) roughly increases with the square of the ion charge, beam ordering effects of highly charged ions were observable with electron cooling at extremely low beam currents $[5,6]$, where density dependent heating mechanisms become negligible.

In the relativistic regime, that will be accessible at FAIR, the situation might be reversed. Electron cooling cannot be readily applied any more, while the laser force principally increases with the third power of the ion energy $[2,7]$. This prediction is based on the fast transition rates in high- $\mathrm{Z}$

\footnotetext{
* Work supported by the German BMBF 06ML183

$\dagger$ www.ha.physik.uni-muenchen.de/uschramm/
}

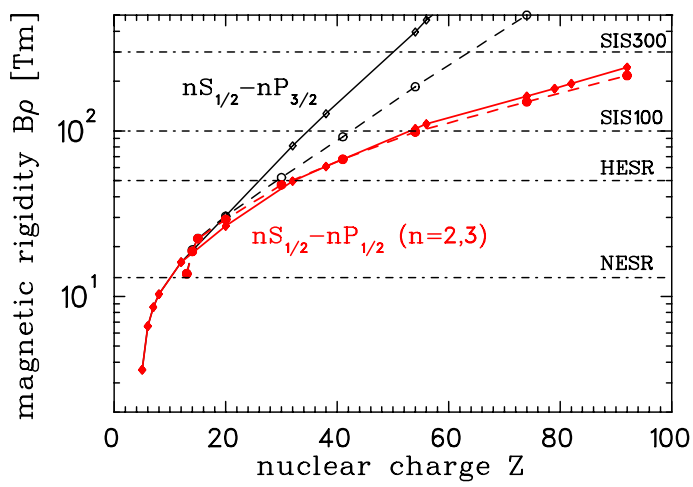

Figure 1: Magnetic rigidity required for the storage of ions at an energy where the transition wavelength of the ground state transitions of Li-like (solid lines, rhombs, $n=2$ ) and Na-like ions (dashed lines, circles, $n=3$ ) are Doppler-shifted into resonance with a counterpropagating laser beam of wavelength $\lambda_{u v}=257 \mathrm{~nm}$.

few electron systems and the relativistically increased momentum transfer in the head-on scattering of laser photons. Moreover, ground-state transitions of all $\mathrm{Li}-$ and most $\mathrm{Na}-$ like heavy ions can be reached (Fig. 1) at the large synchrotrons at FAIR [8, 9] enabling efficient laser excitation for cooling and spectroscopy [10].

\section{LASER COOLING OF $\mathrm{C}^{3+}$ IONS}

As an intermediate step, a test experiment was performed with a $C^{3+}$ ion beam at an energy of $122 \mathrm{MeV} / \mathrm{u}$ ( $\beta=0.47, \gamma=1.13)$ at the heavy ion storage ring ESR (GSI). At this energy both cooling techniques can be applied. For laser cooling, the closed $2 S_{1 / 2}-2 P_{3 / 2}$ transition ( $\lambda_{0}=154.82 \mathrm{~nm}, \tau=3.8 \mathrm{~ns}$ [9]) of the Li-like ions is Doppler-shifted into resonance with the UV-laser line at $\lambda_{\text {laser }} / 2=257.34 \mathrm{~nm}$ [11] when counterpropagating laser and ion beams are used. The decelerating laser force is counteracted by the restoring force of the bucket when the beam is bunched. This technique (sketched in Fig. 2) provides the momentum dependent friction force required for cooling [2] without the need of a copropagating laser beam. Moderate bunching voltages of only few volts were applied at the $10^{t h}$ as well as at the $20^{t h}$ harmonic of the revolution frequency $f_{\text {rev }}=1.295 \mathrm{MHz}$. The bucket depth was determined by the measurement of the synchrotron frequency $f_{\text {sync }} \sim 100 \mathrm{~Hz}(h=10)$ and $f_{\text {sync }} \sim 170 \mathrm{~Hz}$ $(h=20)$ and corresponds to a momentum acceptance of $\Delta p / p \sim 2 \times 10^{-5}$. For an electron-cooled beam this mo- 


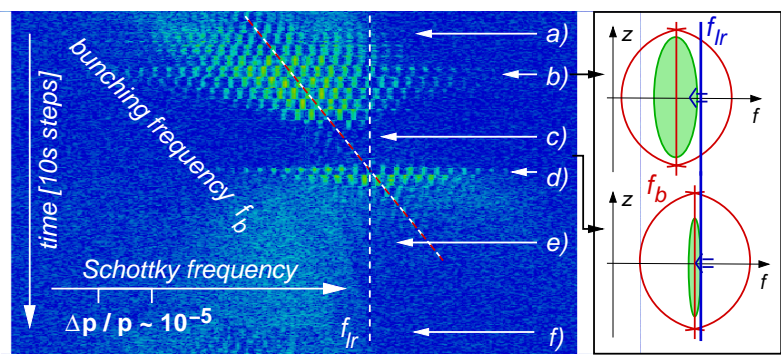

Figure 2: Schottky noise spectrum $\left(47^{t h}\right.$ harmonic, log. color coding) of a laser-cooled bunched $C^{3+}$ beam recorded at scanning bunching frequency $f_{b}$ (diagonal dashed line). The decelerating laser force is resonant with ions at $f_{l r}$ (vertical dashed line). For illustration $f_{b}=h f_{\text {rev }}=10 \times 1.295 \mathrm{MHz}$ is increased in steps of $10 \mathrm{~Hz}$ every $10 \mathrm{~s}$. Starting at a low bunching frequency ions close to the separatrix of the bucket come into resonance with the laser beam and are cooled into the bucket (a). A decreasing number of synchrotron sidebands appears (b), indicating a longitudinally laser-cooled bunch where the ions perform 'incoherent' synchrotron oscillations with $f_{\text {sync }} \sim 100 \mathrm{~Hz}$. The initial envelope corresponds to the momentum acceptance of the bucket $\Delta p / p \sim 2 \times 10^{-5}$. Closer to resonance most sidebands vanish (c) when the beam enters the 'space-charge dominated' regime. Crossing the resonance (d) the synchrotron motion is driven instead of damped. Ions are decelerated (e) out of the bucket until the cycle restarts (f).

mentum spread is reached for a total ion number of few $10^{7}$ (few $10 \mu \mathrm{A}$ ) and is limited by heating due to intra-beam scattering $[2,12]$. The storage-time of the pre-cooled beam amounted to $\tau \sim 450 \mathrm{~s}$ avoiding recombination losses in the electron cooler.

Bunched beam laser cooling means damping of the synchrotron motion by the strong but narrow-band resonant laser force. For the cw-lasers used in the experiment [11], the band-width of the force is determined by the linewidth of the transition corresponding to a (final) momentum spread of $\Delta p / p \approx 5 \times 10^{-8}$. Two schemes exist to overcome this mismatch with the initial momentum distribution. The laser- or the bunching-frequency can be continuously tuned from a value where the laser is resonant with ions at the edge of the bucket to a final frequency close to its center [13]. This scheme is presented in Fig. 2. The second scheme $[14,15]$ relies on the repeated interaction of the ions oscillating in the bucket with the laser tuned to optimum cooling slightly above to the center of the bucket.

For the first scheme, the development of the momentum distribution with increasing cooling strength is depicted in Fig. $3 \mathrm{a}$ as a function of the detuning $\Delta f_{b}$ of the bucket center with respect to the laser resonance. It can be deduced from the envelope of the 'incoherent' side-band spectrum [16] as later discussed with Fig. 4. The development of the spatial distribution, independently measured for a similar ion current using capacitive pick-up devices, is shown in Fig. 3b. Starting at large detuning, the momentum spread
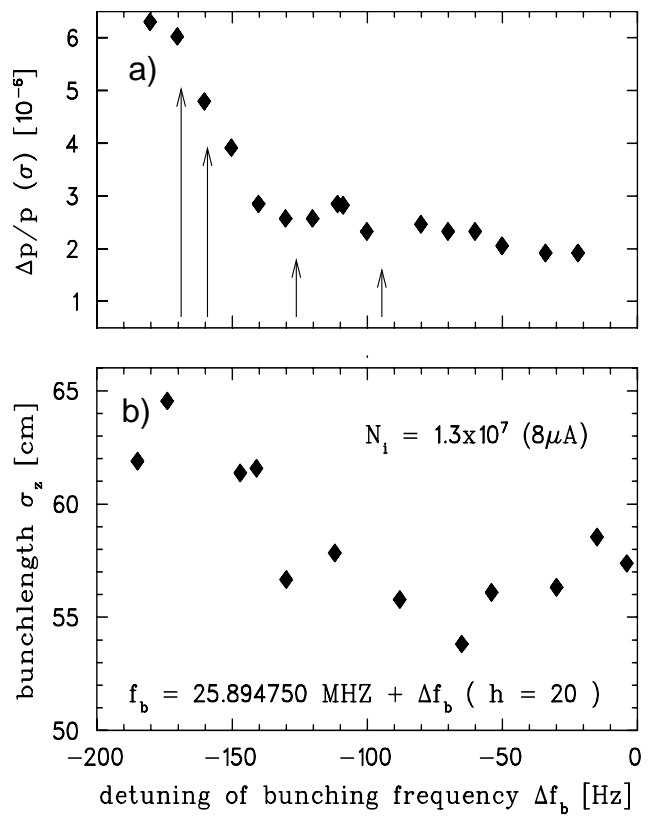

Figure 3: Top (a): Momentum spread deduced from the width of the envelope of the 'incoherent' Schottky sideband spectrum as a function of the detuning $\Delta f_{b}(h=20)$. Arrows indicate the position of the spectra in Fig. 4. Bottom (b): Bunch length (pick-up measurement) limited by the equilibrium length for constant ion density.

as well as the bunch length both decrease with increasing cooling strength. For less detuning than $-100 \mathrm{~Hz}$, corresponding to $\Delta p / p=6 \times 10^{-6}$, the bunch length remains constant and can be reproduced under the assumption of longitudinally 'space-charge dominated' bunches [17] of constant linear density. At most the first synchrotron sidebands remain visible, so that only an upper limit of momentum spread can be deduced beyond this point. Although the Schottky-signal appears to be weaker due to the suppression of the incoherent synchrotron motion, no ions are lost. The integrated pick-up signal and the beam current monitor do not show unexpected ion losses.

In Fig. 4 individual Schottky spectra are plotted that represent the different stages in Fig. 3a. The initial distribution $(a, b)$ shows a symmetric distribution of 'incoherent' synchrotron sidebands with reduced signal strength in the center. This reduction does not correspond to the Bessel-function description of the modulated spectrum for the given momentum spread [16] and might be attributed to the prior laser heating. Little further, satellites become observable on one side of the even side-bands at a frequency separation of $\sim 40 \mathrm{~Hz}$. In the 'space-charge dominated' regime, most sidebands as well as the satellites have vanished, leaving only the carrier and two distinct sidebands (d) at the unaltered spacing of $f_{\text {sync }}=188 \mathrm{~Hz}$. The behavior discussed with Figs. 3 and 4 further depends on the ion current. For higher currents the discontinuity was observed to require less detuning. Sometimes, it became unstable in the way that for further cooling the width again increased, possibly due to an enhanced coupling to the heat-bath of the 


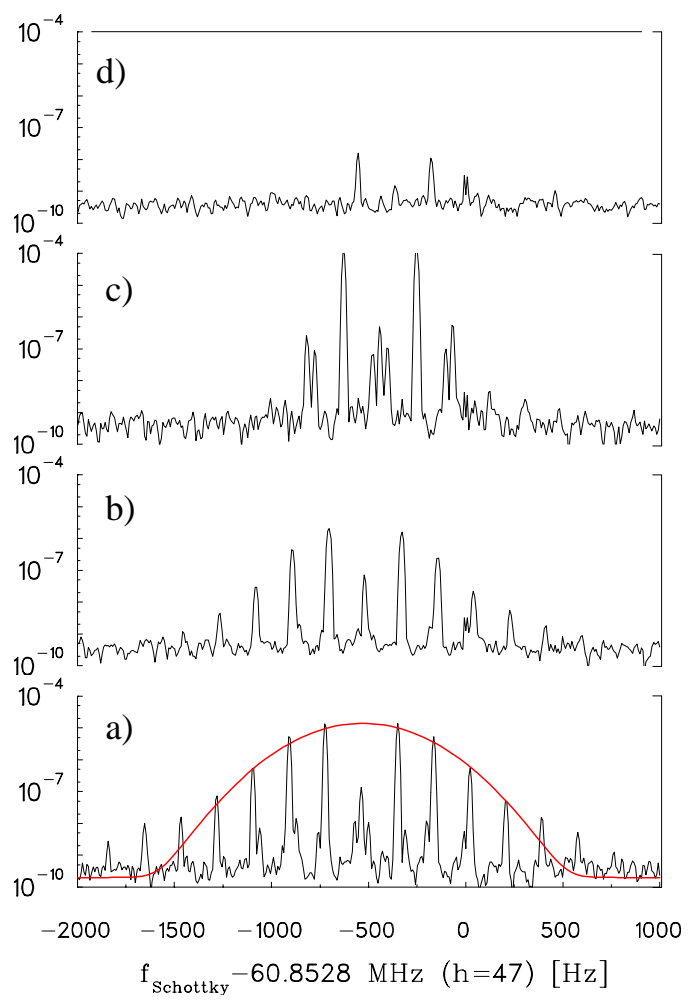

Figure 4: Schottky-noise spectra (same data-set as above) outlined for the detuning marked in Fig. 3b. The red line in (a) represents the Gaussian distribution used for the determination of the momentum spread.

transverse degree of freedom. These effects are presently under investigation as especially indirect transverse cooling is of major interest.

In the 'space-charge dominated' regime the momentum spread cannot be derived from the spatial distribution or the Schottky signal any more. However, laser cooling itself provides a unique diagnostic. Tuning the laser frequency across the Doppler-broadened transition, the momentum distribution can be directly observed via the laser fluorescence signal. In practice, the ion momentum is locally tuned by ramping a drift-tube. The result is depicted in Fig. 5. For a frequency detuning of only $5 \mathrm{~Hz}$ (corresponding to $\Delta p / p=6 \times 10^{-7}$ ) a momentum spread of $\Delta p / p=4 \times 10^{-7}$ is measured for a cold fraction of the beam. It is likely (and with the setup used not resolvable) that part of the beam is about one order of magnitude hotter due to intra-beam scattering and subsequent re-circulation in the bucket. Yet, this beam represents the coldest measured in the ESR. For an ion number of about $1.5 \times 10^{6}$ it corresponds to a plasma parameter of $\Gamma \lesssim 1$ close to the point where ordering phenomena are expected.

Summarizing this test experiment on laser cooling of Lilike carbon ions at $1.47 \mathrm{GeV}$, the full momentum acceptance of the bucket could be laser cooled without prior electron cooling. Yet, the transverse cooling of the injected beam only worked after few seconds of electron precooling. Otherwise, the motion in the different degrees of

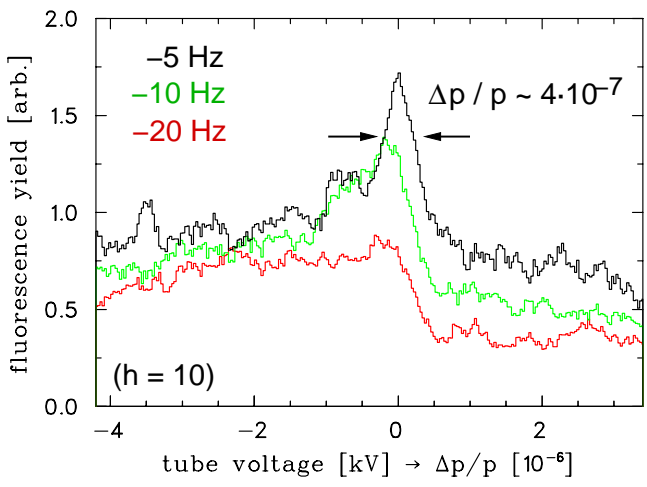

Figure 5: Momentum distribution of laser cooled bunched beam $(h=10,1 \mu \mathrm{A})$ close to minimum detuning, measured via the Doppler effect making use of a local change of the ion momentum inside a drift tube where the fluorescence is recorded, when the tube voltage is ramped.

freedom decoupled and the beam remained transversally hot. For efficient laser cooling of the whole ion bunch, the width of the laser force has to be adapted at least to the momentum range that is given by intra-beam scattering. This is planned to be realized either by a second scanning laser system or by the use of a pulsed laser systems (also providing higher intensities).

\section{REFERENCES}

[1] T. Schätz, et al., Nature (London) 412, 717 (2001), U. Schramm, et al., Phys. Rev. E 66, 036501 (2002)

[2] U. Schramm, D. Habs, Progress in Particle and Nuclear Physics 53, 583 (2004)

[3] U. Schramm, et al., Journal of Physics 36, 561 (2003), "Storage of crystalline beams", PAC'03, Portland, May 2003, TOAA004 (2003)

[4] M. Steck, J. Opt. Soc. Am. B 20, 1016 (2003)

[5] M. Steck, et al., J. Phys. B 36, 991 (2003) and Phys. Rev. Lett. 77, 3803 (1996)

[6] H. Danared, et al., J. Phys. B 36, 1003 (2003)

[7] U. Schramm, et al., Nucl. Instr. Meth. A 532, 348 (2004)

[8] U. Schramm, et al., LoI\#18 FAIR APPA-PAC (2004)

[9] W.R. Johnson, et al., At. Data Nucl. Data Tab. 64, 279 (1996)

[10] U. Schramm. et al., Proc. 'Appl. of Lasers in At. and Nucl. Res.', Poznan, Poland, 2004, Hyperfine Int. in press (2005)

[11] U. Schramm, et al., Hyperfine Int. 115 (1998) 57.

[12] M. Steck et al., this conference (ROPC008)

[13] J.S. Hangst, et al., Phys. Rev. Lett. 74, 4432 (1995)

[14] H.-J. Miesner, et al., Nucl. Instr. Meth. A 383, 634 (1996)

[15] U. Schramm, et al., Phys. Rev. Lett. 87, 184801 (2001)

[16] D. Boussard, CAS, CERN-87/3, 416 (1987), O. BoineFrankenheim, T. Shukla, Phys. Rev. ST AB 8034201 (2005)

[17] T.J.P. Ellison et al., Phys. Rev. Lett. 70, 790 (1993) 